Research Paper

\title{
Interferon-Tau has Antiproliferative effects, Represses the Expression of E6 and E7 Oncogenes, Induces Apoptosis in Cell Lines Transformed with HPV16 and Inhibits Tumor Growth In Vivo
}

\author{
Herbey Oswaldo Padilla-Quirarte, Cesar Trejo-Moreno, Geny Fierros-Zarate, Jhoseline Carnalla \\ Castañeda, Marie Palma-Irizarry, Eva Hernández-Márquez, Ana Isabel Burguete-Garcia, Oscar \\ Peralta-Zaragoza, Vicente Madrid-Marina, Kirvis Torres-Poveda, Victor Hugo Bermúdez-Morales ${ }^{\bowtie}$ \\ Division of Chronic Infection and Cancer, National Institute of Public Health, Av. Universidad 655, Cuernavaca, Morelos. Mexico, 62100. \\ $\triangle$ Corresponding author: Victor Hugo Bermúdez Morales; Email: vbermudez@insp.mx; Tel: (+52-777) 329-30-00; Fax: (+52-777) 317-54-85.
}

(c) Ivyspring International Publisher. Reproduction is permitted for personal, noncommercial use, provided that the article is in whole, unmodified, and properly cited. See http://ivyspring.com/terms for terms and conditions.

Received: 2016.08.10; Accepted: 2016.08.24; Published: 2016.11.05

\begin{abstract}
Interferon tau (IFN- $\tau$ ) is a promising alternative antiviral and immunotherapeutic agent in a wide variety of diseases including infectious, neurodegenerative, autoimmune and cancer due to its low toxicity in comparison with other type I interferon's. The objective of our study was established the effect of the bovine IFN- $\tau$ on human $(\mathrm{SiHa})$ and murine (BMK-16/myc) cells transformed with HPV 16 and evaluates the antitumor effect in a murine tumor model HPV 16 positive. We determine that bovine IFN- $\tau$ has antiproliferative effects, pro-apoptotic activity and induces repression of viral E6 and E7 oncogenes (time- and dose-dependent) on human and murine cells transformed with HPV 16 similar to the effects of IFN- $\beta$. However, IFN- $\tau$ induces greater antiproliferative effect, apoptosis and repression of both oncogenes in BMK-16/myc cells compared to $\mathrm{SiHa}$ cells. The differences were explained by the presence and abundance of the type I interferon receptor (IFNAR) in each cell line. On the other hand, we treated groups of tumor-bearing mice (HPV16 positive) with IFN- $\tau$ and showed the inhibition tumor growth effect in vivo. Our finding indicates that bovine IFN- $\tau$ may be a good candidate for immunotherapy against cervical cancer.
\end{abstract}

Key words: IFN-t; Human papillomavirus; oncogenes; immunotherapy.

\section{Introduction}

Interferon- $\tau$ (IFN- $\tau)$ is a multi-functional cytokine that was first identified as an antiluteolytic protein in ovine pregnancy ${ }^{1}$; it is functionally and structurally similar to the type I IFNs, which include IFNs- $\alpha, \beta$ and $\omega^{2}$. IFN- $\tau$ most resembles IFN- $\omega(\sim 75 \%$ identity) and is similar to IFN- $\alpha$ and IFN- $\beta(\sim 50 \%$ and $\sim 25 \%$ identity, respectively) with non-homology with IFN- $\gamma^{3}$. IFN- $\tau$ has similar biological activity as the classical type I IFNs and binds to the same receptor (IFNAR), but this is not inducible by virus as the classical type I IFNs $(\alpha \text { and } \beta)^{4}$. In addition, has been shown to have antiviral activity in vitro against human immunodeficiency virus (HIV), feline immunodeficiency virus (FIV), ovine lentivirus $(\mathrm{OvLV})$, bovine leukemia virus (BLV), etc, and antiproliferative activity like IFN- $\alpha^{5-7}$. However, IFN- $\tau$ has been demostrated to be less cytotoxic than IFN- $\alpha$, at comparatively high doses in vitro ${ }^{8}$, thus is a cytokine with potential therapeutic.

IFN- $\tau$ has been shown to induce or increase expression of IFN-stimulated genes (ISGs) such as IRF1, ISG15, 2', 5' -oligoadenylate synthetase $(\mathrm{OAS})^{8}$, 
Mx GTPase 1 (Mx-1) ${ }^{9}$, major histocompatibility complex (MHC) class I, and beta-2-microglobulin $(\mathrm{B} 2 \mathrm{M})^{10}$ which are mediated by the Type I IFN signal transduction pathway, which helps explain its related to the antiviral properties. IFN- $\tau$ also has antiproliferative properties similar to those of the classical type I IFNs $(\alpha, \beta \text { and } \omega)^{6}$. IFN- $\tau$ has been shown to have antiproliferative activity on bovine rat and human cells ${ }^{6}$, including amnion epithelium, human lung large cell carcinoma (NCI-H460); human colon adenocarcinoma (DLD-1); human malignant melanoma (SK-MEL-28); human renal adenocarcinoma (ACHN); human promyelocytic leukemia (HL-60); human T cell lymphoma line (H9); human cutaneous $\mathrm{T}$ cell lymphoma (HUT 78) and human breast adenocarcinoma (MCF7) ${ }^{11}$. Although IFN- $\tau$ is a non-human protein, it has activity in human cells, suggesting that it may be useful as a therapeutic agent for cancer and viral diseases.

Particularly IFNs has been widely used in treatment of cervical intraepithelial neoplasia (CIN) and cervical cancer $(\mathrm{CC})^{12,13}$. Down-regulation of main human papillomavirus (HPV) E6 and E7 oncogenes expression has been observed after IFNs treatment ${ }^{14,15}$, but the effect toxicity has been observed. In HPV positives, cervical cancer cells treated with IFN- $\beta$ causes a strong suppression cell proliferation and induces E6 and E7 repression, however, the molecular basis has not been well determined $^{16}$. We propose that IFN- $\tau$ will be an excellent cytokine to CIN and CC treatment by low toxicity. The $\mathrm{CC}$ represent a tumor generated by oncogenic virus, thus is possible evaluates the antitumor effect and antiviral function of IFN-t. In this context, is necessary investigate the ability of IFN- $\tau$ to down regulate the transcription of E6 and E7 oncogenes, and also determine the suppression of cell proliferation and apoptotic effects in vitro as a therapeutic potential to CC. This asseveration will be answer using cell lines HPV transformed with oncogenic papillomavirus that express E6 and E7 oncogenic and compared the effect with a classical type I IFNs.

The main goal of our study was determined the repression of HPV oncogenes (E6/E7) and antiproliferative effects of bovine IFN- $\tau$ on human and murine cells transformed with human papillomavirus (HPV 16) compared to a classical type I IFNs (IFN- $\beta$ ). The inclusion of a murine epithelial cell line transformed with HPV 16, lies in its ability to induces tumors in Balb/c immunocompetent mice, which would allow further evaluation of the antitumor effect of IFN- $\tau$ in vivo. Our results demonstrate that both interferon's (IFNs) inhibit proliferation of the cell lines in vitro, with no differences were detected between IFN- $\beta$ and IFN- $\tau$. However, HPV 16-transformed murine cells (BMK-16/myc) showed greater inhibition of proliferation than the human cells. We also analyzed the effects of both IFNs on the expression of E6 and E7 HPV oncogenes in human and murine cells; the treatment with both IFNs reduced the expression of the transcripts of viral oncogenes E6 and E7 and had pro-apoptotic activity, but the IFN- $\tau$ induced greater repression of both oncogenes in murine BMK-16/myc cells compared to human SiHa cells transformed with HPV 16. These results can be explained in part by the greater detection of receptor of the type I interferon receptor (IFN- $\alpha / \beta R \beta$ ) in BMK-16/myc cells than in $\mathrm{SiHa}$ cells, which could be responsible for the response of IFN-t in BMK-16/myc cells. Thus, our findings show that bovine IFN- $\tau$ may be a good candidate for immunotherapy against cervical cancer.

\section{Materials and Methods}

\section{Cell Culture}

Cell lines transformed with HPV 16 genome: SiHa (human, HPV 16-positive) and BMK-16/myc (murine, HPV 16-positive), WI-38 (human, fetal lung-fibroblast HPV-negative), C-33 A (human, HPV-negative) and HaCaT (human, HPV-negative) were cultured in Dulbecco's Modified Eagle's Medium (DMEM) supplemented with $10 \%$ of fetal bovine serum (FBS), $10 \mu \mathrm{g}$ of ampicillin, $2.5 \%$ of streptomycin, and $2.5 \%$ of amphotericin $\mathrm{B}$ at $37^{\circ} \mathrm{C}$, in $95 \%$ humidity, and $5 \%$ of $\mathrm{CO}_{2}$. SiHa cells were obtained from American Type Culture Collection (ATCC) and BMK-16/myc cells were generously donated by Dr. Sophe Hallez (Université Libre de Bruxelles, Rhode-saint-genese, Belgium) ${ }^{17,18}$. BMK-16/myc cell line was established by co-transformation of baby BALB/C kidney cells with the c-myc gene and the HPV 16 genome, and this cell line produces tumors in immunocompetent mice.

\section{Proliferation Assay}

The proliferation assay was performed via the colorimetric method based on reduction of MTT (3-(4,5-dimethylthiazol-2-yl)-2,5-diphenyltetrazolium bromide). $8 \times 10^{3}$ cells were seeded in 96-well plates. After $24 \mathrm{~h}$ of incubation, the cells were treated with increasing concentrations of IFN- $\tau$ (12.5, 25, 50 and $100 \mathrm{ng} / \mathrm{mL}$ ) (American Research Products) for different amounts of time $(0,24,48$ and $72 \mathrm{~h})$. After each incubation time, $200 \mu \mathrm{L}$ of MTT $(0.456 \mu \mathrm{g} / \mu \mathrm{L}$, Sigma $)$ were added to each well and $4 \mathrm{~h}$ later the crystals were diluted with DMSO and then read in a spectrophotometer at $540 \mathrm{~nm}$ absorbance. The effect of IFN- $\tau$ was compared with treated cells with 
identical conditions of IFN- $\beta$ (Probiomed), with non-treated cells with FBS, and with non-treated cells without FBS as a negative control of cellular proliferation.

\section{Real-Time RT-PCR}

Total cellular RNA was isolated with Tripure from cells treated with $50 \mathrm{ng} / \mathrm{mL}$ of IFN- $\tau$ at $0,24,48$ and $72 \mathrm{~h}$. Afterwards, $1 \mu \mathrm{g}$ of RNA total was reverse transcribed to synthesize cDNA. The PCR reaction was performed with TaqMan probes for E6 and E7 (E6-F5'-GAGCGACCCAGAAAGTTACCA-3', E6-R: 5'-TGTTGCTTGCAGTACACATTCTAATAT, probe E6M2FAM 5'-CAGCTCTGTGCATAACT3'-5NFQ, E7-F:5-CCGGACAGAGCCCATTACAATAT-3, E7-R:5'GAATGTCTACGTGTGTGCTTTGT, probeE7FAM: 5'-CTACGCTTCGGTTGTGCG-35NFQ) and GAPDH (ID:Mm 00484668_m1) as a housekeeping gene (Applied Biosystems). The PCR reaction was carried out using the Sequence Detection System ABI-PRISM 7900 HT (Applied Biosystems). Samples were amplified using the following PCR variables: 1 cycle of $50^{\circ} \mathrm{C}$ for $2 \mathrm{~min}, 1$ cycle of $95^{\circ} \mathrm{C}$ for $10 \mathrm{~min}$, then 40 cycles of $95^{\circ} \mathrm{C}$ for $15 \mathrm{sec}, 60^{\circ} \mathrm{C}$ for $30 \mathrm{sec}$, and $72^{\circ} \mathrm{C}$ for $30 \mathrm{sec}$. Finally, the gene expression analysis was made with the comparative $\mathrm{Ct}$ method through the formula $2-\Delta \Delta \mathrm{Ct}$.

\section{Western Blot assay}

The SiHa and BMK-16/myc cells were lysed in cold RIPA lysis buffer (20mM Tris-HCL, pH 7.4, 10 $\mathrm{mM} \mathrm{NaCl}, 10 \mathrm{mM} \mathrm{KCl}, 3 \mathrm{mM} \mathrm{MgCl} 2,0.5 \%$ triton $\mathrm{X}-100,0.5 \%$ NP-40 and protease inhibitors) by incubating for $30 \mathrm{~min}$ at $4^{\circ} \mathrm{C}$. The proteins were then collected by centrifugation and the concentration was determined using BCA protein assay kit (Pirce, Rockford, 11l., USA), according to the manufacturer's instructions. $50 \mu \mathrm{g}$ of total protein was electrophoresed in SDS-PAGE 10\% and the resolved proteins were transferred to nitrocellulose membranes (Amersham Biosciences, Piscataway, N.J., USA). The membrane was incubated with specific antibodies followed by horseradish peroxidase-conjugated secondary antibody incubation. The protein bands were detected using Pierce ECL Western Blotting substrate (Thermo Scientific). The antibody dilutions used were anti-IFN- $\alpha / \beta R \beta, M-300$ sc-30015 (dilution 1:500) and anti- $\beta$ actin, C-11, sc-1615 (dilution 1:100): Santa Cruz Biotechnology (Santa Cruz, Calif., USA).

\section{Measurement Apoptosis assay}

Apoptosis was determine using the Caspase-Glo 3/7 Assay (G8090, Promega), Briefly, 8x103 cells SiHa and BMK-16/myc were culture in 96-well plates, after
$24 \mathrm{~h}$ of incubation, the cells were treated with 50 $\mathrm{ng} / \mathrm{mL}$ of IFN- $\tau$ (American Research Products) and IFN- $\beta$ (Probiomed) for different amounts of time $(0,24$, 48 and 72h). Equal volume of Caspase-Glo 3/7 reagent was added directly to each well and the plates were incubated at $37^{\circ} \mathrm{C}$ for $1 \mathrm{~h}$. The caspase activity (firefly luciferase) was measured by GloMasx Multi Microplate Luminometer (Promega Madison, WI, USA) using a protocol standard. Data are men \pm SD of three determinations and significant differences.

\section{Tumor inhibition assay}

Preclinical evaluation was carried out in an HPV-16-positive murine tumor model ${ }^{17}$. BALB/c mice at 6 to weeks of age were purchased from Charles River Laboratories, Wilminton, MA, USA. 5x10 BMK-16/myc cells were injected subcutaneously into the back of Balb/c mice at a previously shaved site. Each group of five mice, their tumor volume was measured in $\mathrm{mm}^{3}$ every 3 days $(\mathrm{V}=$ larger diameter $\mathrm{X}$ smaller diameter $\left.{ }^{2}\right) / 2$. Tumor volume of 20 to $30 \mathrm{~mm}^{3}$ was considered time zero and the subsequent effect of IFN- $\tau$ on tumor growth. Each group of mice was treated with $100 \mathrm{ng}$ of IFN- $\tau$ and IFN- $\beta$ administered intra-tumorally in a final volume of $20 \mu \mathrm{l}$ in 1X PBS. Another group of five mice with treated with only $1 \mathrm{X}$ PBS $(20 \mu \mathrm{l})$ and a group without treatment (control). All mice were monitored for a period of 28 days; tumor inhibition was determined by measuring tumor volume $\left(\mathrm{mm}^{3}\right)$ every three days with a digital caliper.

\section{Statistical Analysis}

Data were analyzed with the software GraphPad Prism 5. For the proliferation studies the ANOVA test was performed and student's $t$-test was used for the expression of E6 and E7. To analyzed the difference in tumor volume an ANOVA test was performed. In both cases, a $\mathrm{p}<0.05$ was considered statistically significant.

\section{Results}

\section{IFN-t decreased the proliferation in human and murine HPV 16-transformed cells}

Initially, to examine the effect of IFN- $\tau$ on SiHa and BMK-16/myc cell growth, we analyzed the cell growth inhibition of the cells with different concentrations of IFN- $\tau$ at various time points. MTT assay was used to monitor proliferation in monolayer cultures of replicate cultures of HPV 16-transformed cells and also the effect on proliferative of the IFN- $\tau$ on the cells was similar with the IFN- $\beta$. We observed that the IFN- $\beta$ and IFN- $\tau$ had a clear antiproliferative effect that was dose- and were dose- and timedependent on human and murine HPV 
16-transformed cells (Figures 1 and 2). The BMK-16/myc cell had greater cell growth inhibition by both IFNs than the SiHa cells with percentage reductions of up $81 \%$ and $78 \%$ respectively at the highest dose $(100 \mathrm{ng} / \mathrm{mL})$. However, when non-transformed HPV cells (C-33 A, HaCaT and WI-38) were treated with both IFNs $(100 \mathrm{ng} / \mathrm{mL})$, the
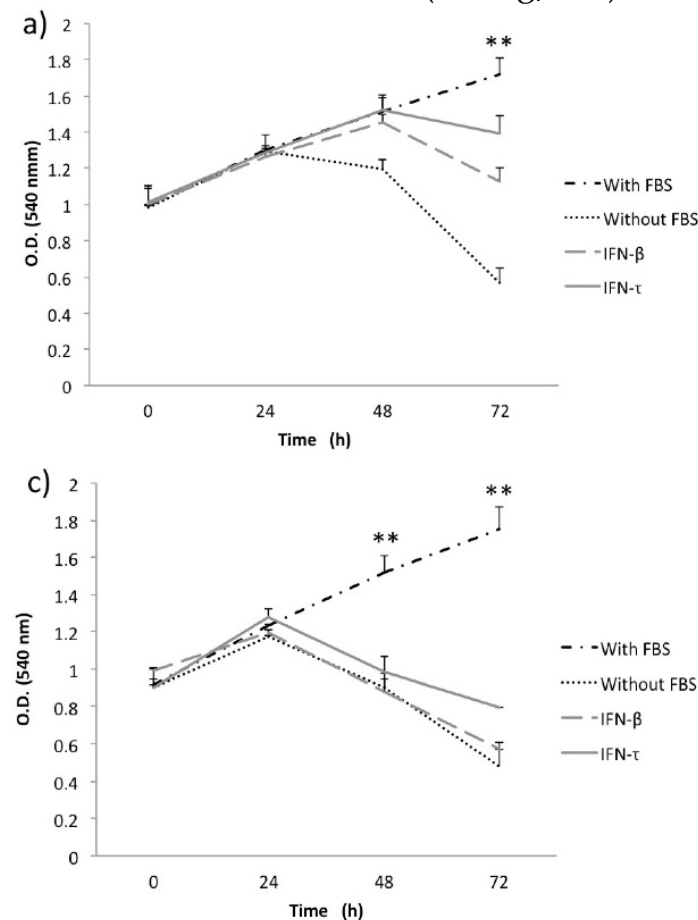

antiproliferative effect was not similarly to the one observed in the HPV 16-transfromed cells (Supplementary Figure 1). Cell growth inhibition was not as evident as in the SiHa and BMK-16/myc cells. The HaCaT and C-33 A cells showed greater cell growth inhibition than the WI-38 cells.
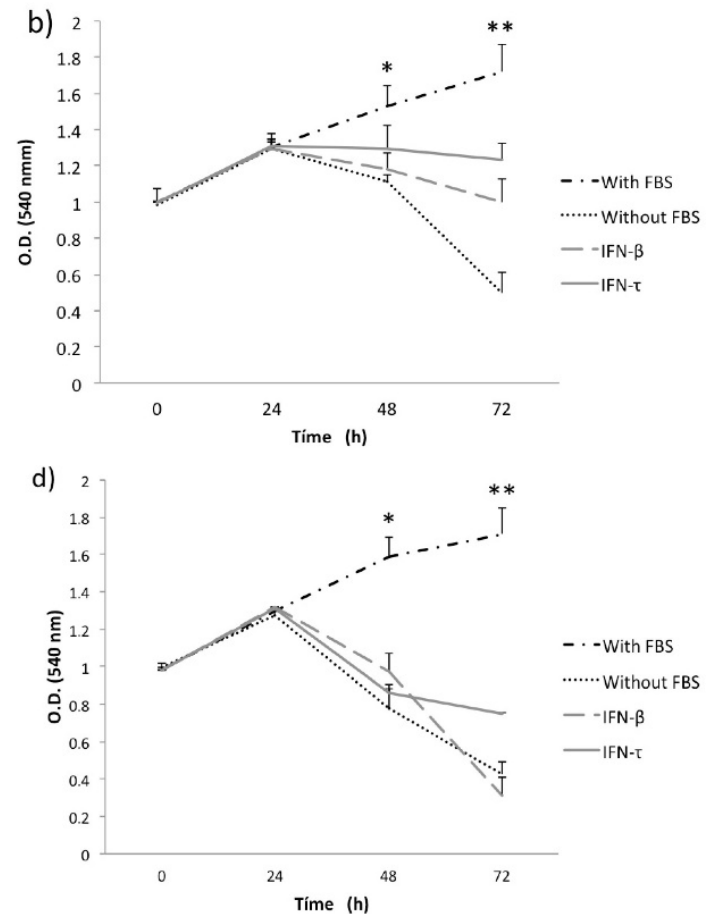

Figure 1. IFN- $\tau$ ovine inhibits the proliferation in SiHa cells. SiHa cells were cultured with various concentration 12.5 (a), 25 (b), 50 (c) and $100 \mathrm{ng} / \mathrm{mL}$ (d) of IFN- $\tau$ or IFN- $\beta$. After the proliferation assays performed with the MTT method and results represent the mean \pm SD of three experimental performed in triplicate. The $P$ values were determined by mean of ANOVA assay with the software GraphPad Prism (*p<0.05; **p<0.001).
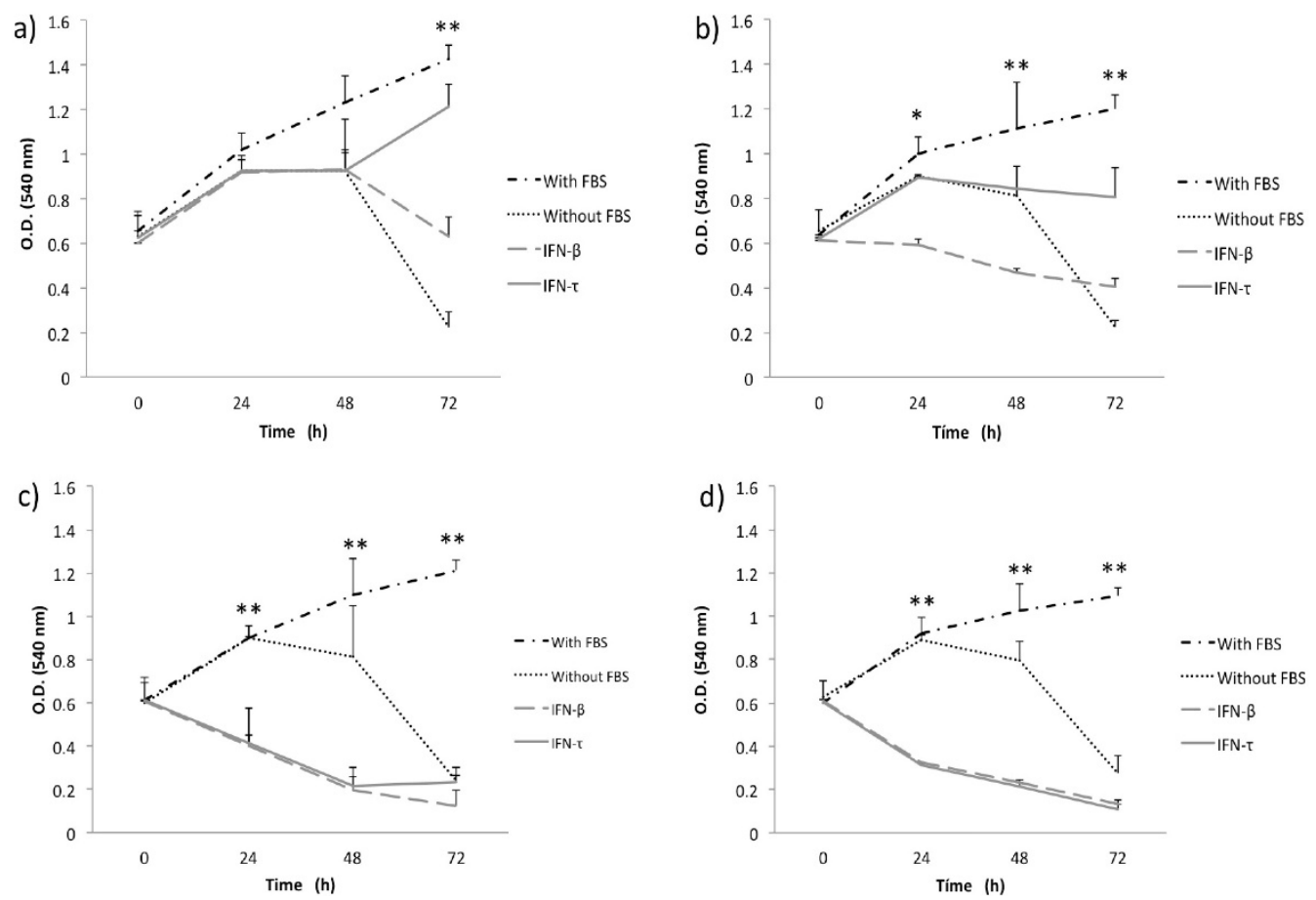

Figure 2. IFN- $\tau$ ovine inhibits the proliferation in BMK-16/myc cells. BMK-16/myc cells were cultured with various concentration 12.5 (a), 25 (b), 50 (c) and $100 \mathrm{ng} / \mathrm{mL}$ (d) of IFN- $\tau$ or IFN- $\beta$. After the proliferation assays performed with the MTT method and results represent the mean \pm SD of three experimental performed in triplicate. The $P$ values were determined by mean of ANOVA assay with the software GraphPad Prism ( $\left.{ }^{*} \mathrm{p}<0.05 ;{ }^{*} \mathrm{p}<0.001\right)$. 
IFN- $\tau$ down regulates the expression of the mRNA of E6 and E7 in SiHa and BMK-16/myc cells

Simultaneously to the antiproliferative effect of IFN-t, we also evaluate the effect of both INFs on the expression of HPV E6 and E7 oncogenes in BMK-16/myc and SiHa cells. Previously we had demonstrated that the BMK-16/myc cell line transformed with HPV 16 constitutively expressed similar levels of E6/E7 viral oncogenes as human cells, such as SiHa cells. The detection of E6 and E7 oncogenes was done by real time RT-PCR one-step. The expression of HPV 16 E6 oncogene in SiHa and BMK-16/myc cells is affected by both IFNs, and this effect is accentuated on the HPV-transformed murine cells, $\mathrm{p}<0.05$ (Figure 3 ). Similar effects were observed for the E7 oncogene, the repression of the mRNA of this viral gene was observed at $48 \mathrm{~h}$ after treatment with IFNs. In both cell lines, we observed reduction in the expression of these genes compared to un-treated cells, but IFN- $\tau$ induces greater repression of both HPV 16 oncogenes in BMK-16/myc cells compared to $\mathrm{SiHa}$ cells, and no differences in repression were detected between the oncogenes in BMK-16/myc cells.

\section{IFN- $\tau$ induces apoptosis in $\mathrm{SiHa}$ and BMK-16/myc cells}

In order to analyze the apoptosis activated by IFN- $\tau$ in malignant cells (SiHa and BMK-16/myc cells), the cell was cultured and treated in presence or absence with $50 \mathrm{ng}$ both IFNs for 24, 48 and 72 hours, then the apoptosis was measure by the activation of effector caspases $3 / 7$. These assays revealed that the IFN- $\tau$ and IFN- $\beta$ display significant difference in the amount of activated caspase 3/7 compared with cell no-treated (Figure 4). We observed an increase in the apoptosis in $\mathrm{SiHa}$ and BMK-16/myc cell by both IFNs and were time-dependent. Seventy-two hours after the treatment, the cells showed significant activation of caspases, inferring cell death by apoptosis. Notability, BMK-16/myc cells had greater significant induction of apoptosis with the INFs, but the IFN- $\tau$ presents a slightly increased induction of apoptosis in relation with IFN- $\beta$.
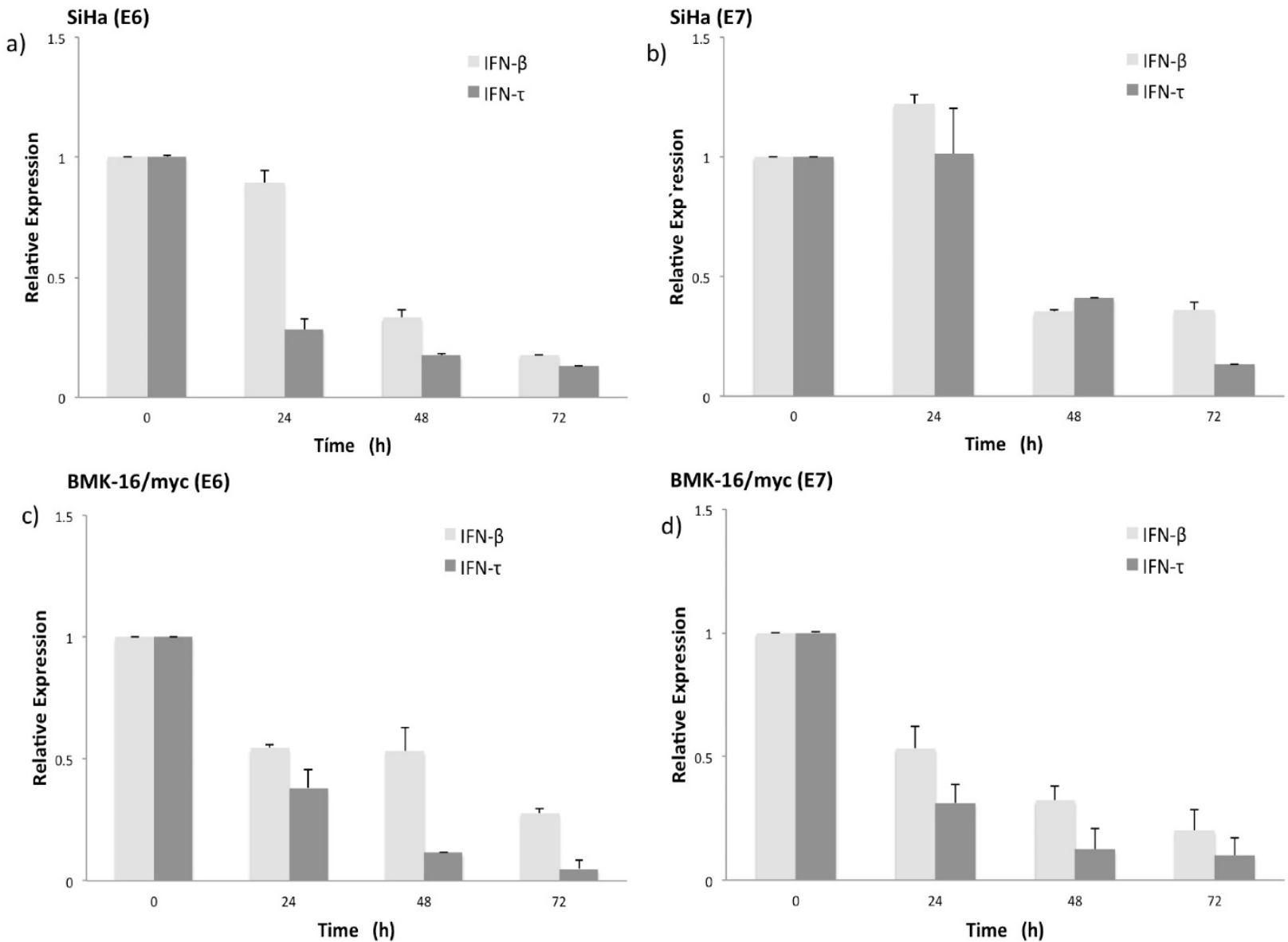

Figure 3. Relative expression levels of the mRNA of HPV oncogenes $E 6$ and $E 7$ in $\mathrm{SiHa}(\mathrm{a}, \mathrm{b})$ and BMK-16/myc cells (c, d) treated with $50 \mathrm{ng} / \mathrm{mL}$ IFN- $\tau$ or IFN- $\beta$. By quantitative real time RT-PCR was detected the HPV 16 E6 and E6 mRNA expression and is presented with a scale of fold change calculated by $2-\Delta \Delta C t$, In order to detect significant differences among the effect of both interferon's a student's t-test was performed $\left({ }^{*} \mathrm{p}<0.05\right)$. 


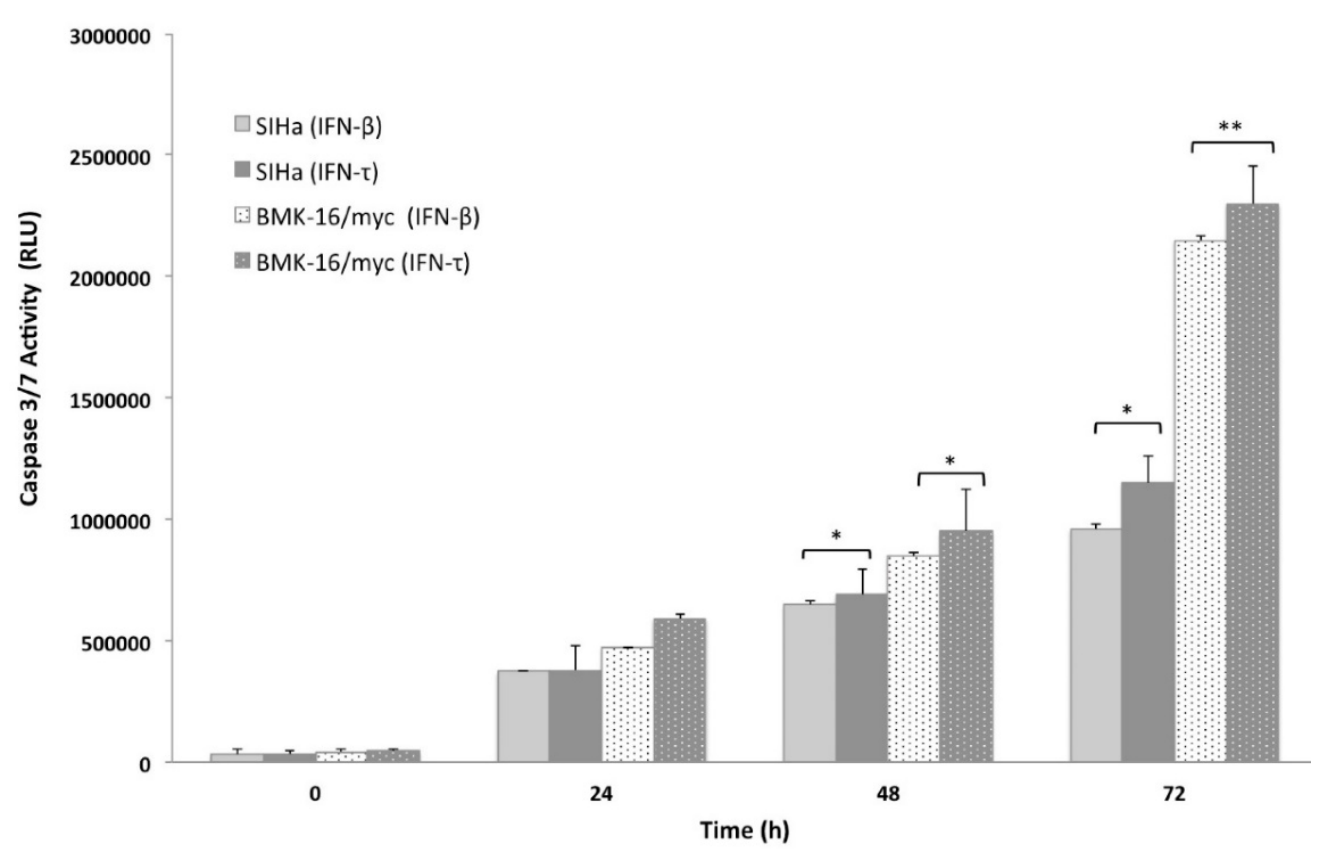

Figure 4. Concentration dependent caspase $3 / 7$ activity in SiHa and BMK-16/myc cells treated with treated with $50 \mathrm{ng} / \mathrm{mL}$ of IFN- $\tau$ or IFN- $\beta$ for 72 hours and then le levels of caspase were measured using Caspase-Glo 3/7 Assay. Columns, the means of two experiments; bar, S.D., 0 untreated control. (*) Indicate $p<0.05$, vs. controls.

A

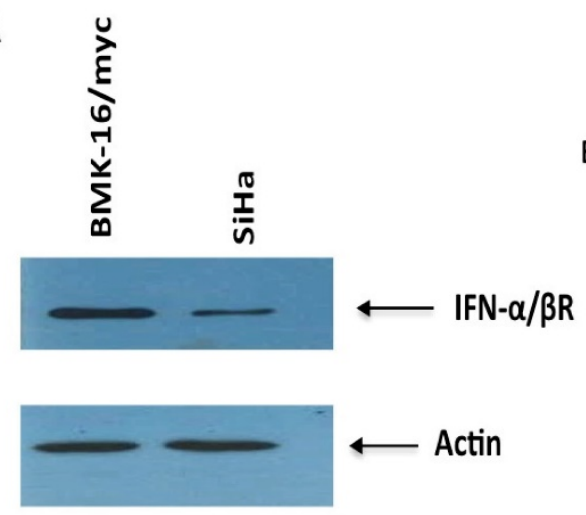

B

BMK-16/myc

$\mathrm{SiHa}$

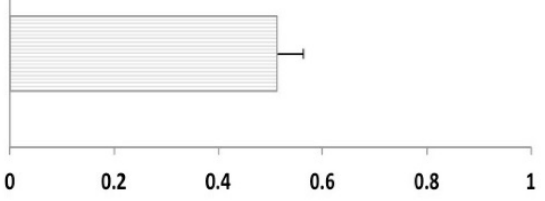

Relative Units of Expression

Figure 5. Detection of receptor beta $(\mathrm{FN}-\alpha / \beta R \beta)$ of type I interferon receptor (IFNAR) by Western blot. Protein isolates of HPV 16 -transformed BMK-16/myc (murine) and SiHa (human) cell line were electrophoresed on SDS-polyacrylamide gels and transferred on nitrocellulose transfer membranes. After a protein-blocking step, blots were incubated with the chicken anti-receptor beta $(F N-\alpha / \beta R \beta)$ of type I IFNAR. After addition of a HRP-conjugated donkey anti-rabitt secondary antibody, bound enzyme was detected with Pierce ECL Western Blotting substrate. Arrow- heads indicate the band corresponding to the $\mathrm{FN}-\alpha / \beta \mathrm{R} \beta(55 \mathrm{kDa})$ and the actin protein $(43 \mathrm{kDa}) \mathrm{was}$ used as a control.

\section{Detection of protein from interferon beta-receptor (IFN- $\alpha / \beta R \beta)$ in SiHa and BMK-16/myc cells}

The differences observed in cell growth inhibition, repression of HPV 16 E6 and E7 oncogenes and activation of apoptosis by the IFNs on human cells and murine HPV 16-transformed cells, will be explained in part by the abundance of receptor of the type I interferon. So, we detected the protein of the type I interferon beta-receptor (IFN- $\alpha / \beta R \beta$ ) and was quantified in each cell line by western blot. We observed clear differences between the cell lines; the protein was detected and stained intensely in BMK-16/myc cells compared to very faint band in SiHa cells, indicating that the receptor is present in differing amount in these cell lines (Figure 5).

\section{IFN-t inhibits tumor growth in vivo}

One week after tumor cell injections of BMK-16/myc cells into the back, palpable tumors formed in the injection site and around 2 weeks the tumor volume was of 20 to $30 \mathrm{~mm}^{3}$. Nine days after the injection of IFN- $\tau$ (one dose), a significant difference in tumor size between the groups (IFN- $\beta$, PBS and control) was observed (Figure 6). The tumor growth was much slower in mice treated with IFN- $\tau$ than the mice groups treated with IFN- $\beta$ and controls $(p>0.05)$. Eighteen days after the treatment with the interferon's, is evident continues tumor growth in the mice group treated with IFN- $\beta$ and the mice treated with IFN-t remains tumor-free mice (Figure 7). 


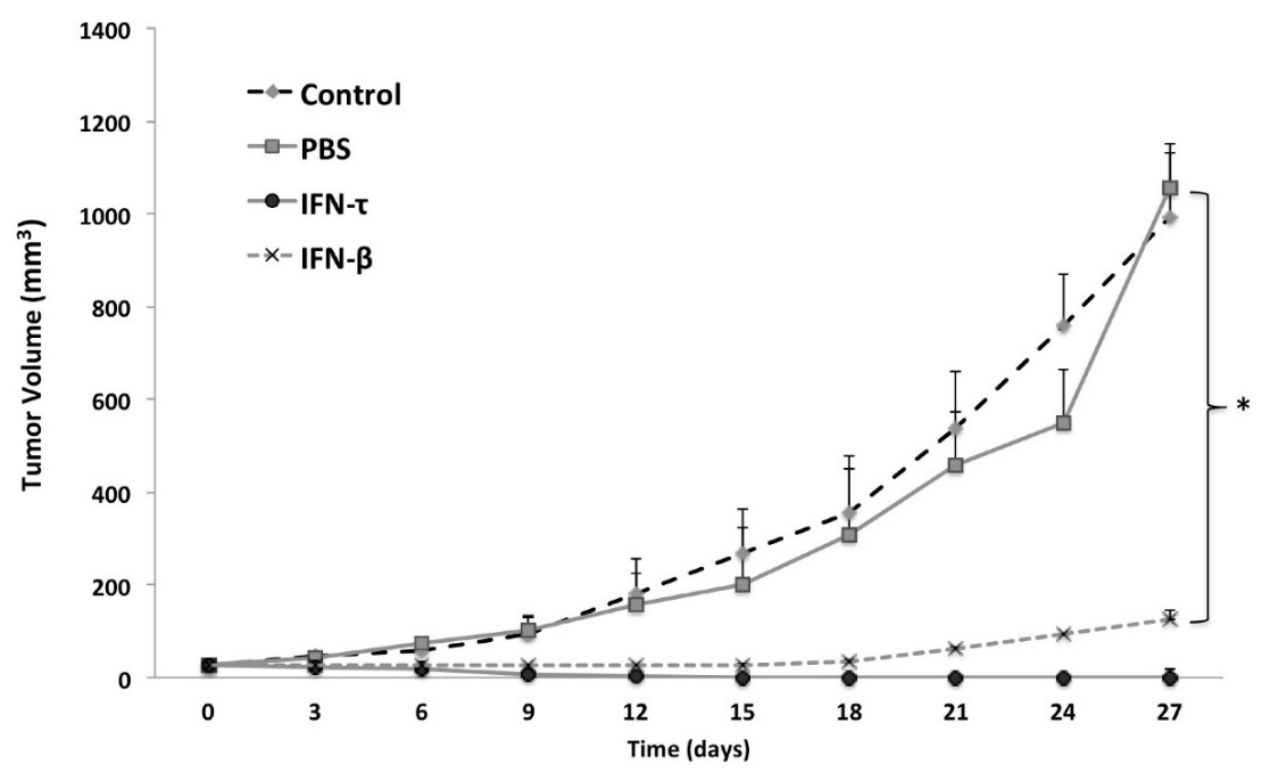

Figure 6. IFN- $\tau$ inhibits tumor growth in vivo. Balb/c mice were inoculated with $5 \times 10^{5}$ BMK-16/myc cells subcutaneously into the back. Tumor growth was monitored with a digital caliper. Mice were treated with $100 \mathrm{ng}$ of IFN- $\tau$ and IFN- $\beta$ administered intra-tumorally after tumor implantation (tumor volume of 20 to 30 mm ${ }^{3}$ ). The average tumor size was compared with of the treated and non-treated tumor. Each tumor model included $n=5$ mice. Error bars indicate SD. Statistically significant differences indicated by asterisks).

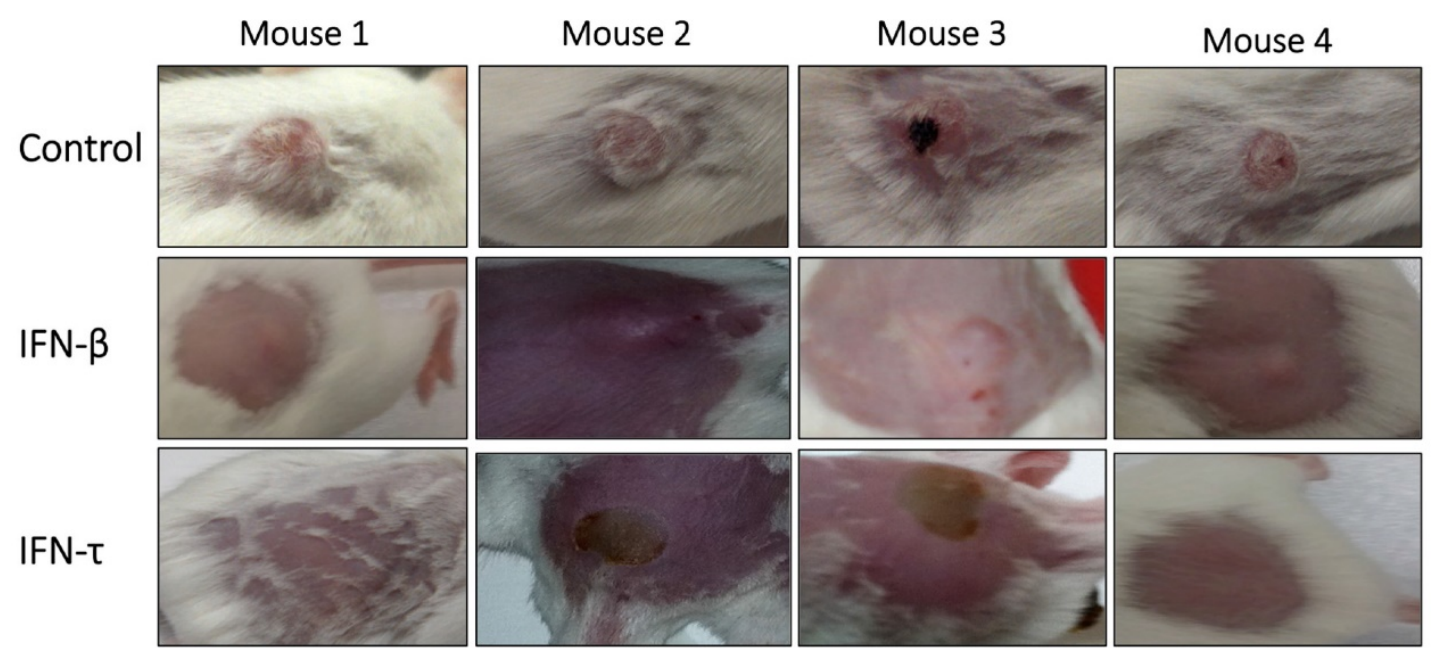

Figure 7. Antitumor activity of IFN- $\tau$ in a tumor model of immunocompetent mice HPV 16 -positive. Tumor-bearing mice were treated with IFN- $\tau$, IFN- $\beta$ ( 100 ng one dose) and control. At the beginning of treatment, the tumor was well developed and growing rapidly, and six days after the treatment with IFN- $\tau$, the tumor began to decrease, and by day 10 only a stain on the epithelial tissue was visible in some mice, which gradually disappeared. Whereas tumor-bearing mice treated with IFN- $\beta$, only partial tumor reduction was observed.

\section{Discussion}

IFN- $\alpha$, IFN- $\beta$ and IFN- $\gamma$ are widely recognized for their antiviral and antiproliferative effects, and these properties have been exploited through their clinical application in the treatment of viral infections or malignant diseases ${ }^{19,20}$. However, these cytokines have dose-dependent toxicity, which limits their range of application in various diseases. Consequently, other classes of IFNs have garnered attention due to their therapeutic potential and comparatively lower cytotoxicity at high doses. IFN- $\tau$ is a promising alternative antiviral and immunotherapeutic agent in a wide variety of diseases including infectious, neurodegenerative, autoimmune and cancer. We are particularly interested in the treatment of viral agents associated with cancer, such as HPV and cervical cancer and investigate the application of IFN- $\tau$, which may be an attractive candidate to replace IFN- $\beta$, another type I interferon, in HPV and other diseases. Therefore we characterized the repression of HPV oncogenes (E6/E7) and antiproliferative effects of bovine IFN- $\tau$ in HPV 16-transformed cell lines in vitro, to further evaluate IFN-t's antitumoral and immunoregulatory propperties on an experimental HPV 16-positive mouse tumor model. 
Our major finding was that bovine IFN- $\tau$ has antiproliferative effects on HPV 16-transformed human and murine cells similar to classical type I IFNs like IFN- $\beta$. The effects were dose- and timedependent and the BMK-16/myc cell line had greater inhibition of cell growth inhibition by both IFNs than did $\mathrm{SiHa}$ cells. In addition, the SiHa and BMK-16/myc cell lines treated with bovine IFN- $\tau$ and human IFN- $\beta$, exhibited reduced expression of HPV oncogenes E6 and E7. However, greater repression of the viral oncogenes was detected in the BMK-16/myc cells and IFN- $\tau$ had greater effects in these cells. These results are consistent with other studies, which have demonstrated that type I IFNs have a cytostatic effect and induce down regulation of HPV oncogene expression in cell lines derived from cervical cancer and other malignancies ${ }^{21-24}$. Investigators have suggested that there are differences in the different cervical carcinoma cell lines, all of which contain integrated HPV but which have distinct biological responses to IFNs. In our investigation, we found differences in inhibition of cell proliferation and viral gene down regulation by IFN- $\tau$ and IFN- $\beta$ between the effect of the in the human and murine epithelial cell lines, despite the fact that both cell lines are transformed with the same HPV type (HPV 16). This effect could be the result of the human and bovine origin of both IFNs and their different effects on the human and murine cells. However, the differences could be explained in part by the presence and abundance of the IFNAR in each cell line. We analyzed the type I interferon receptor beta $(\mathrm{FN}-\alpha / \beta R \beta)$ by western blot in BMK-16/myc and SiHa cells and detected differences between the cells lines with the murine cells showing higher levels of receptor compared to the human cells. This could account for the BMK-16/myc cells being more sensitive to the action of IFNs and particularity IFN- $\tau$. However, we cannot rule out that the IFN- $\tau$ uses other non-classical pathway or binds different receptors with different effects, as has been speculated in the cases of IFN- $\alpha{ }^{25}$. Furthermore, the mechanisms associated to the effects of the IFNs may be different in each cell line and it may dependent on the transformed condition of the cells. When we evaluated IFN- $\tau$ and IFN- $\beta$ on HPV-negatives cells lines, $\mathrm{HaCaT}$ and C-33 A (transformed-human epithelial cells) and WI-38 (non-transformed human fibroblast cells), we detected cell growth inhibition, but not the same as the one observed in HPV-16 transformed cells (without significant differences between both IFNs); the WI 38 cells showed a lower antiproliferative effect. These results are interesting when compared with those observed in HPV 16 transformed epithelial cells ( $\mathrm{SiHa}$ and BMK-16/myc).
The antiproliferative effects shown by the IFNs are greater, this is because they repress the expression of E6/E7-oncogenes, and this event is related to the inhibition of cell growth ${ }^{26}$. The restoration of the normal levels of tumor suppressor proteins p53 and $\mathrm{pRb}$ after repression of the E6 and E7 oncogenes by the IFNs on SiHa and BMK-16/myc is linked to the apoptosis and the suppression of cellular proliferation.

On the other hand, in our tumor model of immunocompetent mice HPV 16-positive, tumor protection was obtained six and eight days after the administration of a single dose of IFN-t. It is possible that the antitumor effect is also associated to the immunoregulatory properties of IFN-ז in vitro, which involves the activation of macrophages 27, the induction of the stimulates granulocyte-macrophage colony-stimulating factor (GMC-CSF) ${ }^{28}$ and the MX gene expression in peripheral blood mononuclear cells $\quad(\mathrm{PBMC})^{29}$. Also, stimulates interferon-gamma-inducible protein $10 \mathrm{kDa}$ (IP10) ${ }^{30}$, ISGs (IRF1, ISG15) ${ }^{8}, \mathrm{OAS}^{8}$ and MHC class $\mathrm{I}^{10}$. Some of these proteins could be involved in inhibition of angiogenesis and could inhibit tumor growth by blocking tumor vascularization linked to the activation of the immune response. The IP10 protein has the ability to chemo-attract tumor-specific T-cells to the tumor site and also to promote the proliferation of these recruited T-cells leading to the tumor regression evaluated in a 4T1-implanted Balb/c mouse model (breast cancer model) ${ }^{31}$. However, perhaps the best immunoregulatory property of IFN- $\tau$ associated with anti-tumoral effect in vivo is that the IFN- $\tau$ it is a potent activator of GM-CSF. Peripheral blood leukocytes stimulated with IFN- $\tau$ produced 1000 times more GM-CSF than endothelial cells ${ }^{28}$. It is quite possible that IFN- $\tau$ favors the activation of $\mathrm{T}$ cells (CD4+ and CD8+ cells) and that this is related to its antitumor effect. GM-CSF has been proved in clinical therapy in refractory recurrent breast and female genital tract carcinoma and the leukocytes seem to be a predictor of response. ${ }^{32}$ GM-CSF is a powerful cytokine that plays a crucial role in the generation of antigen-presenting cells and mononuclear precursors both ex vivo and in vivo 33,34 . The local or systemic administration of GM-CSF alone or in combination with interferons also increases the dendritic cell to the antigen-presenting to establish an intrinsic cellular immunologic response against autologous tumor cell 33-35. Additionally, the antitumor action of IFN- $\tau$ has only been observed in a myeloma tumor model. The RM4/IFN- $\tau$ fusion causes tumor regression, which is mediated by activation of the CD8+ cytotoxic lymphocytes ${ }^{37}$. Thus, the antitumor effects of IFN- $\tau$ in vivo can be explained, at 
least partly, by the repression of the E6 and E7 oncogenes, the inhibition of cell growth and the induction of apoptosis of BMK-16/myc cells, all of which suggests that it played an important role in the suppression of tumor growth in our experimental tumor model. The contribution of our data is the antitumor effect of IFN- $\tau$ on an HPV 16-positive experimental tumor in vivo in immunocompetent mice inoculated with the BMK-16/myc cell line (see Materials and Methods). However, further studies are needed to elucidate the mechanism involved in the immunoregulatory properties of IFN- $\tau$ associated with its antitumor effect in vivo.

In summary, our results demonstrate the repression of HPV oncogenes oncogenes (E6/E7) and antiproliferative effect of IFN- $\tau$ on human and murine cells transformed with HPV 16, as well as, the presence of the type I interferon receptor beta. Furthermore, the IFN- $\tau$ has antiviral and antiproliferative effects on $\mathrm{SiHa}$ cells comparable to IFN- $\beta$. We also demonstrated the antitumor effect of IFN- $\tau$ in a tumor model of HPV 16-positive immunocompetent mice, making IFN- $\tau$ a promising and less cytotoxic alternative to classical type I IFNs for treatment of cervical cancer associated with HPV infection. Further research is necessary to clarify the mechanism by which IFN- $\tau$ exerts these effects on cells, including investigation of type I interferon receptor activation and signal transduction. It will also be necessary to demonstrate IFN- $\tau$ 's immunomudulatory effects in vivo, and efficacy in animal models and ultimately in clinical trials.

\section{Conclusions}

The present study establishes the antiproliferative effect and repression of human papillomavirus (HPV) oncogenes (E6/E7) of bovine IFN- $\tau$ on human (SiHa) and murine (BMK-16/myc) cells transformed with HPV 16. The antiproliferative effect and repressor of viral oncogenes by IFN- $\tau$ was time-and dose-dependent, and IFN- $\tau$ induces greater effect in BMK-16/myc cells compared to SiHa cells. The differences were associated by the abundance of the IFNAR in the BMK-16/myc cells compared to the $\mathrm{SiHa}$ cells. We also demonstrated the antitumor effect of IFN- $\tau$ in a tumor model of HPV 16-positive immunocompetent mice. In conclusions, our results demonstrate that bovine IFN- $\tau$ is a cytokine may be a good candidate for immunotherapy against cervical cancer.

\section{Supplementary Material}

Supplementary figure 1.

http://www.jcancer.org/v07p2231s1.pdf

\section{Acknowledgments}

The funds for this work were obtained from the Mexican government through a Grand National Council of Science and Technology (CONACYT) with file numbers: Conacyt-Basica-2011-168817, ConacytProyectos de Desarrollo Científico para Antender Problemas Nacionales-2013-215484.

\section{Conflicts of Interest}

The authors declare no conflict of interest.

\section{References}

1. Imakawa K. Anthony RV, Kazemi M, et al.: Interferon-like sequence of ovine tropho- blast protein secreted by embryonic trophectoderm. Nature 1987;330:377-379.

2. Sen GC: Viruses and interferons. Annu Rev Microbiol 2001;55:255-281.

3. Roberts RM, Liu L, Alexenko A. New and atypical families of Type 1 interferons in mammals: comparative functions, structures, and evolutionary relationships. Prog Nucleic Acid Res Mol Biol 1997;56:287-325.

4. Chelmońskasoyta A. Interferon tau and its immunobiological role in ruminant reproduction. Arch Immuno Ther Exp (Warsz) 2002;50:47-52.

5. Pontzer $\mathrm{CH}$, Torres BA, Vallet JL, et al. Antiviral activity of the pregnancy recognition hormone ovine trophoblast protein-1. Biochem Biophys Res Commun 1988;152:801-807.

6. Pontzer $\mathrm{CH}$, Bazer FW, Johnson HM: Antiproliferative activity of a pregnancy recognition hormone, ovine trophoblast protein-1. Cancer Res 1991;51:5304-5307

7. Pontzer $\mathrm{CH}$, Yamamoto JK, Bazer FW: Potent anti-feline immunodeficiency virus and anti-human immunodeficiency virus effect of IFN-tau. I Immunol 1997; 158:4351-4357.

8. Chon DT, Bixler S: Interferon-t: Current Applications and Potential in Antiviral Therapy. Interferon Cytokine Res 2010;30:477-485.

9. Song G, Han JH, Spencer TE, Bazer Z: Interferon Tau in ovine uterus. J Anim Sci Technol 2009;51:471-484

10. Choi Y, Johnson GA, Spencer TE, Bazer FW: Pregnancy and interferon tau regulate major histocompatibility complex class I and beta2-microglobulin expression in the ovine uterus. Biol Reprod 2003;68:1703-1710.

11. Johnson FW, Ott HM, Pontzer TL, Van Heeke C. Antitumor therapy using ovine or bovine interferon-tau. 1999. US 5958402 A

12. Baker GE, Tyring SK: Therapeutic approaches to papillomavirus infections. Dermatol Clin 1997;15:331-40.

13. Bonnez W, Oakes D, Bailey-Farchione A: A randomized double-blind trial of parenteral low dose versus high dose interferon- $\beta$ in combination with cryotherapy for treatment of condyloma acuminatum. Antiviral Res 1997;35:41-52.

14. Khan MA Tolleson $\mathrm{WH}$, Gangemi JD, et al. Inhibition of growth, transformation, and expression of human papillomavirus type 16 E7 in human keratinocytes by alphainterferons. J Virol 1993;67(6): 3396-3403.

15. Chiantore MV, Mangino G, Iuliano M, et al.: IFN- $\beta$ antiproliferative effect and miRNA regulation in Human Papilloma Virus E6- and E7-transformed keratinocytes. Cytokine 2015; S1043-4666:30127-7.

16. Zhang $\mathrm{J}, \mathrm{Li} \mathrm{S}$, Yan $\mathrm{Q}$, et al. Interferon- $\beta$ induced microRNA-129-5p down-regulates HPV-18 E6 and E7 viral gene expression by targeting SP1 in cervical cancer cells. PLoS One. 2013:16;e81366.

17. Bermúdez VH, Peralta O, Guzmán E: HPV 16 E2 protein induces apoptosis in human and murine HPV 16 transformed epithelial cells and has antitumoral effects in vivo. Tumour Biol 2009:30:61-72.

18. Crook T, Almond N, Murray A, et al: Constitutive expression of c-myc oncogene confers hormone independence and enhanced growth-factorresponsiveness on cells transformed by human papillomavirus type 16. Proc Natl Acad Sci USA 1989;86:5173-5717.

19. 18Lin FC, Young HA: Interferons: Success in anti-viral immunotherapy. Cytokine Growth Factor Rev 2014;25:369-376.

20. El-Baky NA, Redwan EM: Therapeutic alpha-interferons protein: structure, production, and biosimilar. Prep Biochem Biotechnol 2015;45:109-127.

21. Agarwal C, Hembree JR, Rorke EA, Eckert RL: Interferon and retinoic acid suppress the growth of human papillomavirus type 16 immortalized cervical epithelial cells, but only interferon suppresses the level of the human papillomavirus transforming oncogenes. Cancer Res 1994;54:2108-2112.

22. Kim KY, Blatt L, Taylor MW: The effects of interferon on the expression of human papillomavirus oncogenes. J Gen Virol 2000;81:695-700.

23. Borden EC, Hogan TF, Voelkel JG: Comparative antiproliferative activity in vitro of natural interferons alpha and beta for diploid and transformed human cells. Cancer Res 1982;42:4948-4953.

24. Johnson JA, Hochkeppel HK, Gangemi JD: IFN-tau exhibits potent suppression of human papillomavirus E6/E7 oncoprotein expression. I Interferon Cytokine Res 1999;19:1107-1116. 
25. Zé G, di Marco S, Mouchele E: Domains of interaction between alpha interferon and its receptor components. J Mol Biol 1994;243:245-257.

26. Goodwin EC, DiMario D: Repression of human papillomavirus oncogenes in HeLa cervical carcinoma cells causes the orderlyreactication of dormant tumor suppressor pathways. Proc Natl Acad Sci U S A. 2000;97:12513-12518.

27. Wang B, Goff AK: Interferon-tau stimulates secretion of macrophage migration inhibitory factor from bovine endometrial epithelial cells. Biol Reprod 2003:1690-1696

28. Emond V, Asselin E, Fortier MA: Inteferon-tau stimulates granulocytes-macrophage colony-stimulating factor gene expression in bovine lymphocytes and endometrials stromal cells. Biol Reprod 2000;62: 1728-1737.

29. Yankey SJ, Hicks BA, Carnahan KG: Expression of the antiviral protein MX in peripheral blood mononuclear cells of pregnant and bred, non-pregnant ewes. I Endocrinol 2001;170:R7-R11.

30. Bagaoka K, Sakai A, Nojima A: A chemokine interferon (IFN)-gamma-inducible protein $10 \mathrm{kDa}$ is stimulated by IFN-tau and recruits immune cells in the ovine endometrium. Biol Reprod 2003;68:1413-1421.

31. Taslimi Y, Zahedifard F, Habibzadeh S, et al.: Antitumor effect of IP-10 by using two different approaches: Live delivery system and gene therapy. J Breast Cancer 2016;19:34-44.

32. Kurbacher CM, Kurbacher JA, Cramer EM, et al.: Continuous low-dose GM-CSF as salvage therapy in refractory recurrent. Oncol 2000;4:23-26.

33. Weiss AJ, Lackman RD: A comparison of human G-CSF and human GM-CSF given concurrently with anti-cancer chemotherapy. Oncol Rep 2002;9:945-950.

34. Chang DZ, Lomazow W, Joy Somberg C, et al: Granulocyte-macrophage colony stimulating factor: An adjuvant for cancer vaccines. Hematol 2004;9:207-215.

35. Basak SK, Harui A, Stolina M, et al: Increased dendritic cell number and function following continuous in vivo infusion of granulocyte macrophage-colony-stimulating factor and interleukin-4. Blood 2002;99:2869-2879.

36. Pinedo HM, Buter J, Luykx-de Bakker SA, et al: Extended neoadjuvant chemotherapy in locally advanced breast cancer combined with GM-CSF: Effect on tumour-draining lymph node dendritic cells. Eur J Cancer 2003:39:1061-1067.

37. Qui Y, Moyana T, Chen Y, et al. Characterization of anti-tumor immunity derived from the inoculation of myeloma cells secreting the fusion protein RMA/IFN-tau. Hum Antibodies Hybrodomas 1996;7:21-26. 